\title{
General Output Feedback Stabilization for Fractional Order Systems: An LMI Approach
}

\author{
Yiheng Wei, ${ }^{1}$ Hamid Reza Karimi, ${ }^{2}$ Shu Liang, ${ }^{1,3}$ Qing Gao,, ${ }^{1,4}$ and Yong Wang ${ }^{1}$ \\ ${ }^{1}$ Department of Automation, University of Science and Technology of China, Hefei 230027, China \\ ${ }^{2}$ Department of Engineering, Faculty of Engineering and Science, University of Agder, 4898 Grimstad, Norway \\ ${ }^{3}$ College of Engineering, University of North Carolina Charlotte, Charlotte, NC 28223, USA \\ ${ }^{4}$ Department of Mechanical and Biomedical Engineering, City University of Hong Kong, Kowloon Tong 999077, Hong Kong
}

Correspondence should be addressed to Yong Wang; yongwang@ustc.edu.cn

Received 3 November 2013; Accepted 4 January 2014; Published 20 February 2014

Academic Editor: Peng Shi

Copyright (C) 2014 Yiheng Wei et al. This is an open access article distributed under the Creative Commons Attribution License, which permits unrestricted use, distribution, and reproduction in any medium, provided the original work is properly cited.

\begin{abstract}
This paper is concerned with the problem of general output feedback stabilization for fractional order linear time-invariant (FOLTI) systems with the fractional commensurate order $0<\alpha<2$. The objective is to design suitable output feedback controllers that guarantee the stability of the resulting closed-loop systems. Based on the slack variable method and our previous stability criteria, some new results in the form of linear matrix inequality (LMI) are developed to the static and dynamic output feedback controllers synthesis for the FO-LTI system with $0<\alpha<1$. Furthermore, the results are extended to stabilize the FO-LTI systems with $1 \leq \alpha<2$. Finally, robust output feedback control is discussed. Numerical examples are given to illustrate the effectiveness of the proposed design methods.
\end{abstract}

\section{Introduction}

In recent years, fractional order systems (FOSs) have attracted considerable attention from control community, since many engineering plants and processes cannot be described concisely and precisely without the introduction of fractional order calculus [1-6]. Due to the tremendous efforts devoted by researchers, a number of valuable results on stability analysis [7-9] and controller synthesis [10-13] of FOSs have been reported in the literature.

Since it is usually not possible or practical to sense all the states and feed them back, it is practically important and theoretically appealing to stabilize systems by output feedback controller (OFC) [14-18]. Linear matrix inequality (LMI) is one of the most effective and efficient tools in controller design and a great deal of LMI-based methods of OFC design have been proposed over the last decade. Generally, these methods can be broadly classified into three categories: iterative algorithm $[19,20]$, singular value decomposition (SVD) method [21-24], and slack variable method [25, 26].

Paradoxically, only few studies deal with OFC design for FOSs. One finds some existing results as presented in
[27-30] only. Based on SVD method, [27] designs the OFC for a type of FOS with time delay. Nevertheless, the SVD method is inherently conservative, particularly for such a large number of decision variables. Reference [28] designs an $H_{\infty}$ static OFC which is also based on SVD method, but the stable region reduced repeatedly in the transformation process. Reference [29] studies the FOS stabilization problem based on its approximation model, which does not face the original fractional order systems directly. Reference [30] gives sufficient conditions for static OFC which can stabilize the FOS with the order $1 \leq \alpha<2$. Nonetheless, there is no discussion about $0<\alpha<1$ and dynamic OFC. Furthermore, the original free decision matrix variables $G_{11}, G_{12}, G_{21}$, and $G_{22}$ are limited to $G_{11}=G_{22}=Z$ and $G_{12}=G_{21}=0$, which shall cause an increase in conservatism.

This motivates us to adopt our previous stability criteria [9] in output feedback controller synthesis for FOLTI systems, which shall make the resulting controller less conservative and more applicable in practice. Preferably, the new method is applicable for all the fractional order $0<\alpha<$ 2. In addition, the output feedback stabilization of uncertain FOS is discussed in the paper. 
Section 2 is devoted to some background materials and the main problem. Based on our stability criteria for FO-LTI systems $(0<\alpha<1)$ and the slack variable method, the main results are presented in Section 3. In Section 4, some numerical simulations are provided to illustrate the validity of the proposed approach. Conclusions are given in Section 5.

\section{Problem Formulation and Preliminaries}

Consider the following FO-LTI system:

$$
\begin{gathered}
\mathscr{D}^{\alpha} x(t)=A x(t)+B u(t), \\
y(t)=C x(t),
\end{gathered}
$$

where the order $0<\alpha<2 ; x(t) \in \mathbb{R}^{n}, u(t) \in \mathbb{R}^{m}$, and $y(t) \in$ $\mathbb{R}^{q}$ are the system state, the control input, and the measurable output, respectively; the system matrices $A, B$, and $C$ are the constant real matrices with appropriate dimensions.

We make the usual assumptions that the pair $\{A, B\}$ is controllable and the pair $\{A, C\}$ is observable, which guarantee the existence of real matrices $K_{0}$ and $L_{0}$ such that $A+B K_{0}$ and $A+L_{0} C$ are stable. Thus one may find a real matrix $K$ satisfying the stability of $A+B K C$.

The following Caputo definition is adopted for fractional derivatives of order $\alpha$ for function $f(t)$ :

$$
\begin{aligned}
\mathscr{D}^{\alpha} f(t)= & \frac{1}{\Gamma(m-\alpha)} \\
& \times \int_{0}^{t}(t-\tau)^{m-\alpha-1} f^{(m)}(\tau) \mathrm{d} \tau,
\end{aligned}
$$

where the fractional order $m-1<\alpha \leq m, m \in \mathbb{N}$, and the Gamma function $\Gamma(x)=\int_{0}^{\infty} e^{-t} t^{x-1} \mathrm{~d} t$.

In this paper, the following general OFC is considered:

$$
\begin{gathered}
\mathscr{D}^{\alpha} z(t)=A_{u} z(t)+B_{u} y(t), \\
u(t)=C_{u} z(t)+D_{u} y(t),
\end{gathered}
$$

where $z(t) \in \mathbb{R}^{p}$ is the controller state variable.

Set $A_{u}=0, B_{u}=0, C_{u}=0$, and $D_{u}=K$; the static OFC is derived:

$$
u(t)=K y(t) .
$$

Set $A_{u}=N, B_{u}=M, C_{u}=I$, and $D_{u}=0$; one can obtain the following dynamic OFC:

$$
\mathscr{D}^{\alpha} u(t)=M y(t)+N u(t) .
$$

This paper aims at finding the proper condition so that the resulting closed-loop system is asymptotically stable with the three types of OFCs. For this purpose, the following lemmas are first introduced.

Lemma 1 (see [25]). Let $\Phi, a$, and $b$ be given matrices with appropriate dimensions; then

$$
\begin{gathered}
\Phi<0, \\
\Phi+\operatorname{sym}\left(a b^{T}\right)<0
\end{gathered}
$$

hold if and only if there exists an appropriate dimension matrix G which satisfies

$$
\left[\begin{array}{cc}
\Phi & a+b G^{T} \\
\star & -\operatorname{sym}(G)
\end{array}\right]<0
$$

where the operator sym $(G)$ represents $G+G^{T}$ and $\star$ stands for the symmetrical part matrix; for example,

$$
\left[\begin{array}{cc}
A & B \\
\star & C
\end{array}\right]=\left[\begin{array}{cc}
A & B \\
B^{T} & C
\end{array}\right] .
$$

Proof. Without loss of generality, we assume that $\Phi \in \mathbb{R}^{n \times n}$, $a, b \in \mathbb{R}^{n \times m}$, and $G \in \mathbb{R}^{m \times m}$. Set $X=\left[\begin{array}{cc}\Phi & a \\ \star & 0\end{array}\right], Y=G, P=$ $\left[\begin{array}{ll}0 & I_{m \times n}\end{array}\right]$, and $Q=\left[b^{T}-I_{m}\right]$; then $(7)$ can be transformed into

$$
X+\operatorname{sym}\left(P^{T} Y Q\right)<0
$$

Select $P_{\perp}=\left[\begin{array}{c}I_{n} \\ 0_{m \times n}\end{array}\right]$ and $Q_{\perp}=\left[\begin{array}{c}I_{n} \\ b^{T}\end{array}\right]$, using the projection lemma in [31], and one gets the equivalent LMIs:

$$
\begin{gathered}
P_{\perp}^{T} X P_{\perp}=\Phi<0, \\
Q_{\perp}^{T} X Q_{\perp}=\Phi+\operatorname{sym}\left(a b^{T}\right)<0 .
\end{gathered}
$$

This establishes the proof.

Lemma 2 (see [9]). The $n$-dimensional system $D^{\alpha} x(t)=$ $A x(t)$ with the order $0<\alpha<1$ is asymptotically stable if and only if there exists a matrix $P \in \mathbb{P}^{n \times n}$, such that

$$
\operatorname{sym}(A P)<0,
$$

where $k=\tan \pi \alpha / 2$ and

$$
\mathbb{P}^{n \times n} \triangleq\left\{k X+Y: X, Y \in \mathbb{R}^{n \times n},\left[\begin{array}{cc}
X & Y \\
-Y & X
\end{array}\right]>0\right\}
$$

Remark 3. The $n$-dimensional system $D^{\alpha} x(t)=A x(t)$ with the order $0<\alpha<1$ is asymptotically stable if and only if there exists a matrix $Q \in \mathbb{P}^{n \times n}$, such that

$$
\operatorname{sym}\left(Q^{T} A\right)<0
$$

Proof. Since $A$ and $A^{T}$ have the same set of eigenvalues, the systems $\mathscr{D}^{\alpha} x(t)=A x(t)$ and $\mathscr{D}^{\alpha} x(t)=A^{T} x(t)$ have the same stability.

As a result, $\exists P \in \mathbb{P}^{n \times n}, \operatorname{sym}(A P)<0$ is equivalent to

$$
\exists Q \in \mathbb{P}^{n \times n}, \quad \operatorname{sym}\left(A^{T} Q\right)<0 .
$$

Because

$$
\operatorname{sym}\left(A^{T} Q\right)=\operatorname{sym}\left(Q^{T} A\right),
$$

one completes the proof of Remark 3. 


\section{Main Results}

\subsection{Static Output Feedback Control}

Theorem 4. Design the controller (4) for the system (1) with $0<\alpha<1$; the corresponding closed-loop control system is asymptotically stable, if there exist matrices $Z \in \mathbb{P}^{n \times n}, G \in$ $\mathbb{R}^{m \times m}$, and $H \in \mathbb{R}^{m \times q}$, such that

$$
\left[\begin{array}{cc}
\Xi & Z^{T} B+C^{T} H^{T}-K_{0}^{T} G^{T} \\
\star & -\operatorname{sym}(G)
\end{array}\right]<0
$$

is feasible, and the controller gain is given by

$$
K=G^{-1} H \text {, }
$$

where $\Xi=\operatorname{sym}\left(Z^{T} A+Z^{T} B K_{0}\right)$, and $K_{0}$ is an additional initialization matrix, which is derived from $K_{0}=Q P^{-1}$. The matrices $P \in \mathbb{P}^{n \times n}$ and $Q \in \mathbb{R}^{m \times n}$ satisfy the following $L M I$ :

$$
\operatorname{sym}(A P+B Q)<0 \text {. }
$$

Proof. First, we design a virtual state feedback controller $u(t)=K_{0} x(t)$ for the system in (1), which yields

$$
\mathscr{D}^{\alpha} x(t)=\left(A+B K_{0}\right) x(t) .
$$

By using Lemma 2, one obtains that the system (19) is asymptotically stable if and only if there exists $P \in \mathbb{P}^{n \times n}$, such that

$$
\operatorname{sym}\left(A P+B K_{0} P\right)<0 .
$$

Define $Q=K_{0} P$; one can easily get (18) from (20).

The use of Remark 3 with $Z_{1} \in \mathbb{P}^{n \times n}$ yields

$$
\operatorname{sym}\left(Z_{1}^{T} A+Z_{1}^{T} B K_{0}\right)<0 .
$$

Hereafter, considering the actual output feedback controller $u(t)=K y(t)$, then the resulting closed-loop dynamic system can be described as

$$
D^{\alpha} x(t)=(A+B K C) x(t) .
$$

From Remark 3, one can obtain that the system (22) is asymptotically stable if and only if there exists a matrix $Z_{2} \in \mathbb{P}^{n \times n}$, such that

$$
\operatorname{sym}\left(Z_{2}^{T} A+Z_{2}^{T} B K C\right)<0 .
$$

Owing to the existence of the nonlinear terms $Z_{2}^{T} B K C$, inequality (23) is not an LMI. For the purpose of using the MATLAB LMI toolbox to solve the matrix inequality, we need to linearize the matrix inequality.

Suppose that $Z_{1}=Z_{2}=Z$, which is just the reason why the theorem has conservatism, such that

$$
\begin{aligned}
\operatorname{sym} & \left(Z_{2}^{T} A+Z_{2}^{T} B K C\right) \\
& =\operatorname{sym}\left(Z^{T} A+Z^{T} B K_{0}+Z^{T} B K C-Z^{T} B K_{0}\right) \\
& =\Xi+\operatorname{sym}\left(a b^{T}\right) \\
& <0,
\end{aligned}
$$

where $\Xi<0, a=Z^{T} B$, and $b=C^{T} K^{T}-K_{0}^{T}$.

Based on Lemma 1, (16) and (24) are equivalent. This establishes Theorem 4.
3.2. Dynamic Output Feedback Control I. Under the control of (5), if we define the augmented state $\bar{x}(t)=$ $\left[\begin{array}{ll}x^{T}(t) & u^{T}(t)\end{array}\right]^{T}$, then the related closed-loop control system can be rewritten as

$$
\mathscr{D}^{\alpha} \bar{x}(t)=(\bar{A}+\bar{B} K \bar{C}) \bar{x}(t)
$$

where

$$
\begin{gathered}
{[\bar{A}|\bar{B}| \bar{C}]=\left[\begin{array}{cc|c|cc}
A & B & 0_{n \times m} & C & 0_{q \times m} \\
0_{m \times n} & 0_{m} & I_{m} & 0_{m \times n} & I_{m}
\end{array}\right],} \\
K=[M \mid N] .
\end{gathered}
$$

Theorem 5. $\{\bar{A}, \bar{B}\}$ is controllable, $\{\bar{A}, \bar{C}\}$ is observable.

Proof. According to [1], $\{A, B\}$ is controllable, if and only if the controllability matrix $M_{C}$ is full row rank, where $M_{C}=$ $\left[\begin{array}{llll}B & A B & \cdots & A^{n-1} B\end{array}\right] .\{A, C\}$ is observable, if and only if the observability matrix $M_{O}$ is full column rank, where $M_{O}^{T}=$ $\left[\begin{array}{llll}C^{T} & A^{T} C^{T} & \cdots & A^{(n-1) T} C^{T}\end{array}\right]$.

As a result, the controllability matrix $\bar{M}_{C}$ related to $\{\bar{A}, \bar{B}\}$ can be described as

$$
\begin{aligned}
\bar{M}_{C} & =\left[\begin{array}{lllll}
\bar{B} & \bar{A} & \bar{B} & \ldots & \bar{A}^{n+m-1} \bar{B}
\end{array}\right] \\
& =\left[\begin{array}{cccc}
0_{n \times m} & B & \cdots & A^{n+m-2} B \\
I_{m} & 0_{m} & \cdots & 0_{m}
\end{array}\right],
\end{aligned}
$$

which implies that

$$
\begin{aligned}
& \operatorname{rank}\left(\bar{M}_{C}\right) \\
& \quad=\operatorname{rank}\left(I_{m}\right)+\operatorname{rank}\left(\left[\begin{array}{llll}
B & A B & \cdots & A^{n+m-2} B
\end{array}\right]\right) \\
& \quad \geq \operatorname{rank}\left(I_{m}\right)+\operatorname{rank}\left(\left[\begin{array}{llll}
B & A B & \cdots & A^{n-1} B
\end{array}\right]\right) \\
& \quad=m+n .
\end{aligned}
$$

By virtue of $\bar{M}_{C} \in \mathbb{R}^{(n+m) \times(n+m) m}$,

$$
\begin{aligned}
\operatorname{rank}\left(\bar{M}_{C}\right) & \leq \min (m+n,(m+n) m) \\
& =m+n .
\end{aligned}
$$

All of these stated above lead up to the following:

$$
\operatorname{rank}\left(\bar{M}_{C}\right)=m+n
$$

In other words, $\{\bar{A}, \bar{B}\}$ is controllable.

The corresponding observability matrix $\bar{M}_{O}$ satisfies

$$
\begin{aligned}
\bar{M}_{O}^{T} & =\left[\begin{array}{llll}
\bar{C}^{T} & \bar{A}^{T} \bar{C}^{T} & \cdots & \bar{A}^{(n+m-1) T} \bar{C}^{T}
\end{array}\right] \\
& =\left[\begin{array}{ccccc}
C^{T} & 0_{n \times m} & \cdots & A^{(n+m-1) T} C^{T} & 0_{n \times m} \\
0_{n \times q} & I_{m} & \cdots & B^{T} A^{(n+m-2) T} C^{T} & 0_{m}
\end{array}\right],
\end{aligned}
$$


which implies that

$$
\begin{aligned}
& \operatorname{rank}\left(\bar{M}_{O}\right) \\
& \quad=\operatorname{rank}\left(I_{m}\right)+\operatorname{rank}\left(\left[\begin{array}{llll}
C^{T} & A^{T} C^{T} & \cdots & A^{(n+m-1) T} C^{T}
\end{array}\right]\right) \\
& \quad \geq \operatorname{rank}\left(I_{m}\right)+\operatorname{rank}\left(\left[\begin{array}{llll}
C^{T} & A^{T} C^{T} & \cdots & A^{(n-1) T} C^{T}
\end{array}\right]\right) \\
& \quad=m+n .
\end{aligned}
$$

Since $\bar{M}_{O} \in \mathbb{R}^{(n+m) m \times(n+m)}$, one has

$$
\begin{aligned}
\operatorname{rank}\left(\bar{M}_{\mathrm{O}}\right) & \leq \min (m+n,(m+n) m) \\
& =m+n .
\end{aligned}
$$

Proceeding forward, one has

$$
\operatorname{rank}\left(\bar{M}_{\mathrm{O}}^{T}\right)=m+n .
$$

Consequently, $\{\bar{A}, \bar{C}\}$ is observable. Thus, Theorem 5 has been proved completely.

Theorem 6. Design the controller (5) for system (1) with $0<$ $\alpha<1$; if there exist matrices $Z \in \mathbb{P}^{(n+m) \times(n+m)}, G \in \mathbb{R}^{m \times m}$, and $H \in \mathbb{R}^{m \times(m+q)}$, such that

$$
\left[\begin{array}{cc}
\Xi & Z^{T} \bar{B}+\bar{C}^{T} H^{T}-K_{0}^{T} G^{T} \\
\star & -\operatorname{sym}(G)
\end{array}\right]<0
$$

is feasible, then the resulting closed-loop control system in (25) is asymptotically stabilizable by the output feedback controller

$$
K=G^{-1} H \text {, }
$$

where $\Xi=\operatorname{sym}\left(Z^{T} \bar{A}+Z^{T} \bar{B} K_{0}\right)$, and $K_{0}$ is an additional initialization matrix, which is derived from $K_{0}=Q P^{-1}$. The matrices $P \in \mathbb{P}^{(n+m) \times(n+m)}$ and $Q \in \mathbb{R}^{m \times n}$ satisfy the following LMI:

$$
\operatorname{sym}(\bar{A} P+\bar{B} Q)<0 .
$$

3.3. Dynamic Output Feedback Control II. Substituting the controller (3) into system (1) and defining the augmented state $\bar{x}(t)=\left[\begin{array}{ll}x^{T}(t) & z^{T}(t)\end{array}\right]^{T}$, then the related closed-loop control system can be rewritten as

$$
\mathscr{D}^{\alpha} \bar{x}(t)=(\bar{A}+\bar{B} K \bar{C}) \bar{x}(t),
$$

where

$$
\begin{gathered}
{[\bar{A}|\bar{B}| \bar{C}]=\left[\begin{array}{ccccccc}
A & 0_{n \times p} & 0_{n \times p} & B & 0_{p \times n} & I_{p} \\
0_{p \times n} & 0_{p} & I_{p} & 0_{p \times m} & C & 0_{q \times p}
\end{array}\right],} \\
K=\left[\begin{array}{c|c}
A_{u} & B_{u} \\
\hline C_{u} & D_{u}
\end{array}\right] .
\end{gathered}
$$

Remark 7. In a manner similar to the proof of Theorem 5, one gets that $\{\bar{A}, \bar{B}\}$ is controllable and $\{\bar{A}, \bar{C}\}$ is observable.

Theorem 8. The system (38) with $0<\alpha<1$ is asymptotically stable, if there exist matrices $Z \in \mathbb{P}^{(n+p) \times(n+p)}, G \in$ $\mathbb{R}^{(m+p) \times(m+p)}$, and $H \in \mathbb{R}^{(m+p) \times(q+p)}$, such that

$$
\left[\begin{array}{cc}
\Xi & Z^{T} \bar{B}+\bar{C}^{T} H^{T}-K_{0}^{T} G^{T} \\
\star & -\operatorname{sym}(G)
\end{array}\right]<0
$$

is feasible, and the controller satisfies

$$
K=G^{-1} H \text {, }
$$

where $\Xi=\operatorname{sym}\left(Z^{T} \bar{A}+Z^{T} \bar{B} K_{0}\right)$, and $K_{0}$ is introduced here as an additional initialization parameter matrix derived from $K_{0}=$ $Q P^{-1}$. The matrices $P \in \mathbb{P}^{(n+p) \times(n+p)}$ and $Q \in \mathbb{R}^{(m+p) \times(q+p)}$ satisfy the following LMI:

$$
\operatorname{sym}(\bar{A} P+\bar{B} Q)<0 .
$$

Remark 9. In order to avoid getting the special result of Theorem 4 or 6 , we set $\operatorname{sym}\left(H_{A} H H_{B}\right)>0$, where $H_{A}$ and $H_{B}$ can be chosen as needed.

Remark 10. Theorems 4,6 , and 8 consider the stability problem of the systems in (22), (25), and (38), respectively. One can observe that there are more decision variables in the LMIs in Theorem 6 or 8 than in Theorem 4, which shall increase the computational complexity to solve those LMIs in the former. Of course, since those theorems are just solving convex feasibility problems, and meanwhile the dimensions $m, n$, and $q$ are of limited practical magnitude, there shall not be a computational burden.

3.4. Output Feedback Control with $1 \leq \alpha<2$. For the case of $1 \leq \alpha<2$, if we define $\widetilde{\alpha}=0.5 \alpha$ and $\widetilde{x}(t)=$ $\left[x^{T}(t) \mathscr{D}^{\widetilde{\alpha}} x^{T}(t)\right]^{T}$, thus the equivalent system with $0<\tilde{\alpha}<1$ can be derived as

$$
\begin{gathered}
\mathscr{D}^{\tilde{\alpha}} \tilde{x}(t)=\widetilde{A} \tilde{x}(t)+\widetilde{B} u(t), \\
y(t)=\widetilde{C} \widetilde{x}(t),
\end{gathered}
$$

where

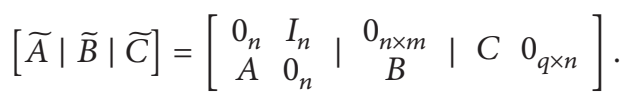

Remark 11. Similar to Theorem 5 , one gets that $\{\widetilde{A}, \widetilde{B}\}$ is controllable and $\{\widetilde{A}, \widetilde{C}\}$ is observable.

Theorem 12. Design the controller (4) for system (1) with $1 \leq$ $\alpha<2$; the related closed-loop control system is asymptotically stable, if there exist matrices $Z \in \mathbb{P}^{2 n \times 2 n}, G \in \mathbb{R}^{m \times m}$, and $H \in$ $\mathbb{R}^{m \times 2 q}$, such that

$$
\left[\begin{array}{cc}
\Xi & Z^{T} \widetilde{B}+\widetilde{C}^{T} H^{T}-K_{0}^{T} G^{T} \\
\star & -\operatorname{sym}(G)
\end{array}\right]<0,
$$


is feasible, and the controller gain is given by

$$
K=G^{-1} H \text {, }
$$

where $\Xi=\operatorname{sym}\left(Z^{T} \widetilde{A}+Z^{T} \widetilde{B} K_{0}\right)$, and $K_{0}$ is an additional initialization matrix, which is derived from $K_{0}=Q P^{-1}$. The matrices $P \in \mathbb{P}^{2 n \times 2 n}$ and $Q \in \mathbb{R}^{m \times 2 n}$ satisfy the following $L M I$ :

$$
\operatorname{sym}(\widetilde{A} P+\widetilde{B} Q)<0 .
$$

Remark 13. Because the way of how to get the controller in Theorem 4 is very similar to that of Theorems 6,8 , and 12 , therefore the proof of the latter three has been omitted.

Remark 14. Both Theorems 5 and 2.3 in [30] focus on designing the controller (4) for system (1) with the order $1<\alpha<2$. There are $m^{2}+2 m q+2 m n+8 n^{2}$ decision variables and $m+4 n$ inequalities in Theorem 12 that need to be solved. At the same time, Theorem 2.3 needs $m^{2}+m q+m n+7 n^{2}+2 n$ decision variables and $m+6 n$ inequalities. Our approach may need more decision variables since some decision variables in Theorem 2.3 are set to be equal or zeros by force, which may become more conservative. In addition, our approach needs less inequalities than that in Theorem 2.3, which shall reduce computational burden.

Remark 15. In analogy to the above mentioned case with $0<\alpha<1$, we can easily get the other stabilization criterion controlled by (3) or (5). Considering expanding the $\alpha$ th order system to $0.5 \alpha$ th order system before or after substituting the controller (3) or (5) into it, we can get $\alpha$ th or $0.5 \alpha$ th controller design criterion.

3.5. Robust Output Feedback Control. Consider the system (1) where $0<\alpha<1$ is uncertain, and the system matrices $A$ and $B$ can be described as

$$
\begin{gathered}
A=A_{0}+\Delta A, \\
B=B_{0}+\Delta B,
\end{gathered}
$$

where $A_{0}$ and $B_{0}$ are the constant matrices and the uncertain terms $\Delta A$ and $\Delta B$ are given by

$$
\left[\begin{array}{ll}
\Delta A & \Delta B
\end{array}\right]=D F(t)\left[\begin{array}{ll}
E_{A} & E_{B}
\end{array}\right]
$$

herein $D, E_{A}$, and $E_{B}$ are constant matrices; the unknown variable matrix $F(t) \in \mathbb{R}^{r \times s}$ satisfies $F^{T}(t) F(t) \leq I_{s}$.

Theorem 16. Design the controller (4) for the system (1) with conditions in (48); the corresponding closed-loop control system is asymptotically stable for all admissible uncertainties, if there exist matrices $Z \in \mathbb{P}^{n \times n}, G \in \mathbb{R}^{m \times m}, H \in \mathbb{R}^{m \times q}$, and a set of positive scalars $\varepsilon_{1}, \varepsilon_{2}, \varepsilon_{3}$, such that

$$
\left[\begin{array}{ccccc}
\Xi_{1} & \Xi_{2} & Z^{T} D & Z^{T} D & Z^{T} D \\
& \Xi_{3} & 0 & 0 & 0 \\
& & -\varepsilon_{1} I_{r} & 0 & 0 \\
& & & -\varepsilon_{2} I_{r} & 0 \\
\star & & & & -\varepsilon_{3} I_{r}
\end{array}\right]<0
$$

is feasible, and the controller gain is given by

$$
K=G^{-1} H \text {, }
$$

where

$$
\begin{aligned}
\Xi_{1}= & \operatorname{sym}\left(Z^{T} A_{0}+Z^{T} B_{0} K_{0}\right)+\varepsilon_{1} E_{A}^{T} E_{A} \\
& +\varepsilon_{2} K_{0}^{T} E_{B}^{T} E_{B} K_{0}, \\
\Xi_{2}= & Z^{T} B_{0}+C^{T} H^{T}-K_{0}^{T} G^{T}, \\
\Xi_{3}= & -\operatorname{sym}(G)+\varepsilon_{3} E_{B}^{T} E_{B} .
\end{aligned}
$$

$K_{0}$ is an additional initialization matrix, which is derived from $K_{0}=Q P^{-1}$. The matrices $P \in \mathbb{P}^{n \times n}, Q \in \mathbb{R}^{m \times n}$, and positive scalars $\lambda_{1}, \lambda_{2}$ satisfy the following LMI:

$$
\left[\begin{array}{ccc}
\Phi & P^{T} E_{A}^{T} & Q^{T} E_{B}^{T} \\
& -\lambda_{1} I_{s} & 0 \\
\star & & -\lambda_{2} I_{s}
\end{array}\right]<0,
$$

where

$$
\Phi=\operatorname{sym}\left(A_{0} P+B_{0} Q\right)+\left(\lambda_{1}+\lambda_{2}\right) D D^{T} .
$$

Remark 17. By using the similar approach in Theorem 4, the theorem can be easily derived, wherefore the proof is omitted.

Remark 18. Analogously, the robust dynamic OFC in exact (3) or (5) with the order $0<\alpha<2$ can be obtained.

\section{Illustrative Examples}

All the numerical examples illustrated in this paper are implemented via the piecewise numerical approximation algorithm. For more information about the algorithm one can refer to [29].

Example 1. Consider the system as follows:

$$
\begin{gathered}
\mathscr{D}^{\alpha} x(t)=\left[\begin{array}{cc}
2.5 & -1 \\
1 & 1
\end{array}\right] x(t)+\left[\begin{array}{l}
1 \\
0
\end{array}\right] u(t), \\
y(t)=\left[\begin{array}{ll}
1 & 1
\end{array}\right] x(t) .
\end{gathered}
$$

It is completely controllable and observable. One gets that the eigenvalues of the system matrix are $1.7500+0.6614 i$ and $1.7500-0.6614 i$, which are denoted by EV0. Thereby, the original system with $\alpha=0.6$ is unstable. If applying the method in Theorems 4,6 , and 8 to design OFCs, using the MATLAB LMI toolbox, one can get the following feasible OFCs:

$$
\begin{gathered}
u(t)=-3.6338 y(t) \\
\mathscr{D}^{0.6} u(t)=-20.7748 y(t)-6.008 u(t) \\
\mathscr{D}^{0.6} z(t)=-\left[\begin{array}{ll}
1.4154 & 0.2302 \\
0.2302 & 1.4154
\end{array}\right] z(t)+\left[\begin{array}{l}
0.2183 \\
0.2183
\end{array}\right] y(t), \\
u(t)=\left[\begin{array}{ll}
1.1802 & 1.1802
\end{array}\right] z(t)-4.6427 y(t)
\end{gathered}
$$




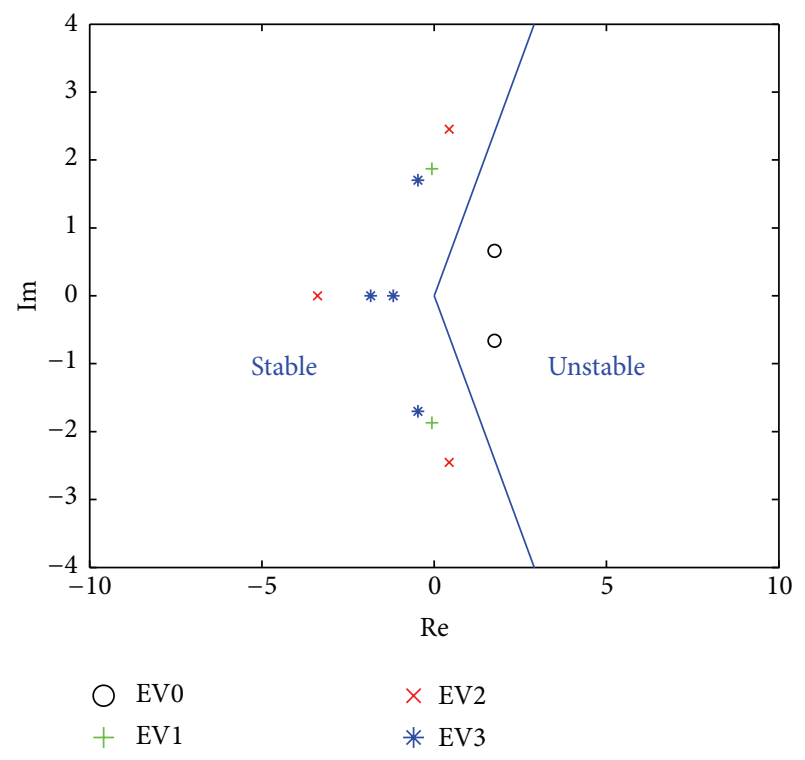

FIGURE 1: Eigenvalues distribution areas of Example 1.

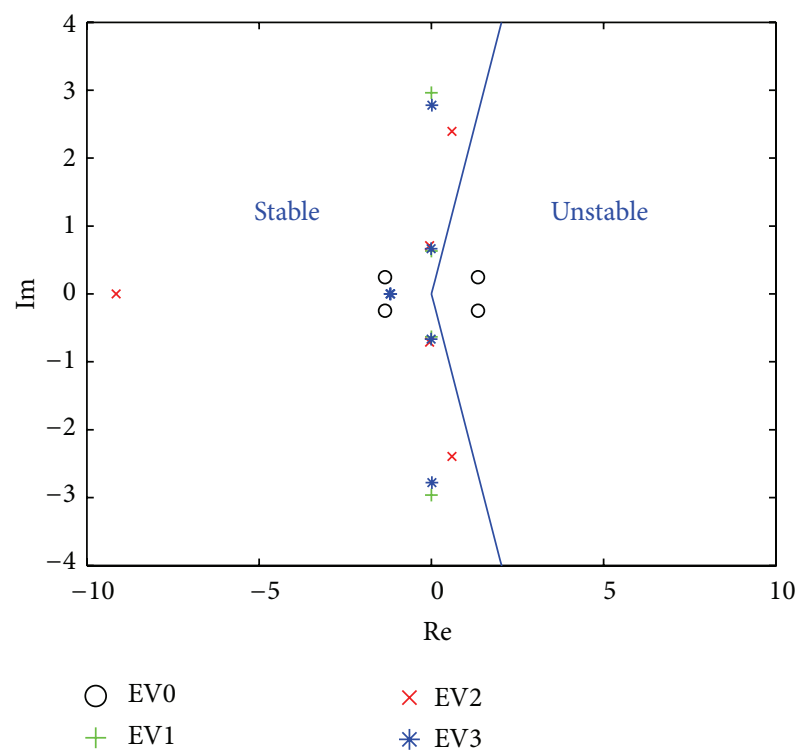

FIgURE 2: Eigenvalues distribution areas of Example 2.

If one uses EV1, EV2, and EV3 to represent the eigenvalues of the closed-loop control system matrices which are controlled by the three aforementioned controller, respectively, then the distribution of those eigenvalues in the complex plane is shown in Figure 1.

Example 2. Consider the system in (55) with $\alpha=1.4$.

According to the method in (43), one gets the equivalent system with 0.7 th order. Based on Remark 15, applying

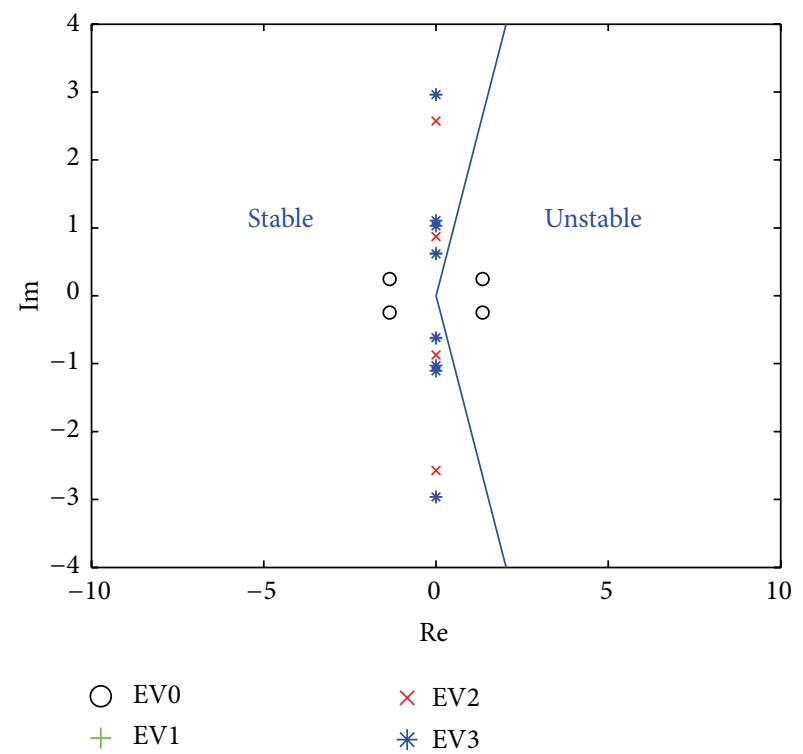

FIGURE 3: Eigenvalues distribution areas of Example 3.

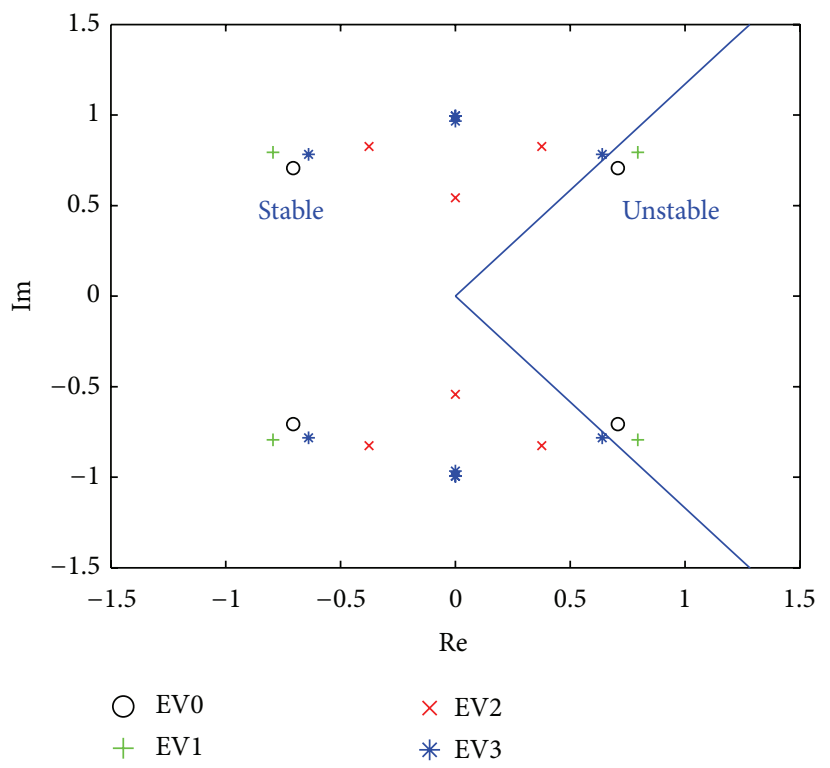

FIGURE 4: Eigenvalues distribution areas of Example 4.

the approaches in Theorems 4, 6, and 8, one can get the following feasible OFCs:

$$
\begin{gathered}
u(t)=-12.6764 y(t) \\
\mathscr{D}^{0.7} u(t)=-87.3660 y(t)-8.0559 u(t) \\
\mathscr{D}^{0.7} z(t)=-\left[\begin{array}{ll}
1.1767 & 0.0004 \\
0.0004 & 1.1767
\end{array}\right] z(t)-\left[\begin{array}{l}
0.0839 \\
0.0839
\end{array}\right] y(t), \\
u(t)=\left[\begin{array}{ll}
1.853 & 1.853
\end{array}\right] z(t)-11.6274 y(t) .
\end{gathered}
$$

The same as Example 1, one obtains the eigenvalues distribution of those equivalent closed-loop control systems in the complex plane as shown in Figure 2. 


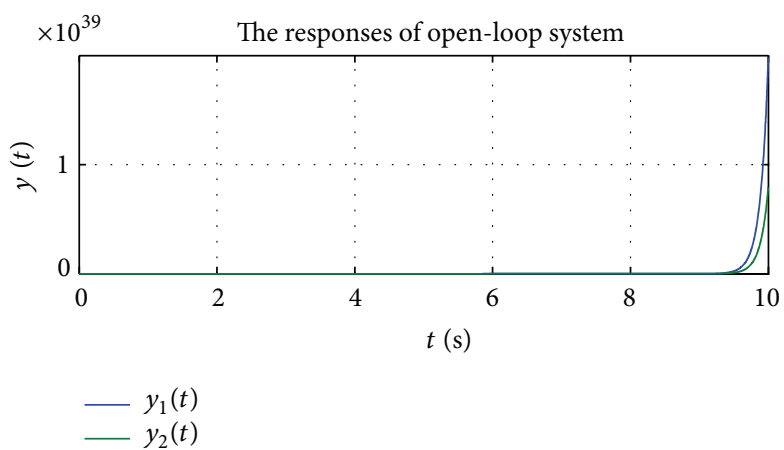

(a)

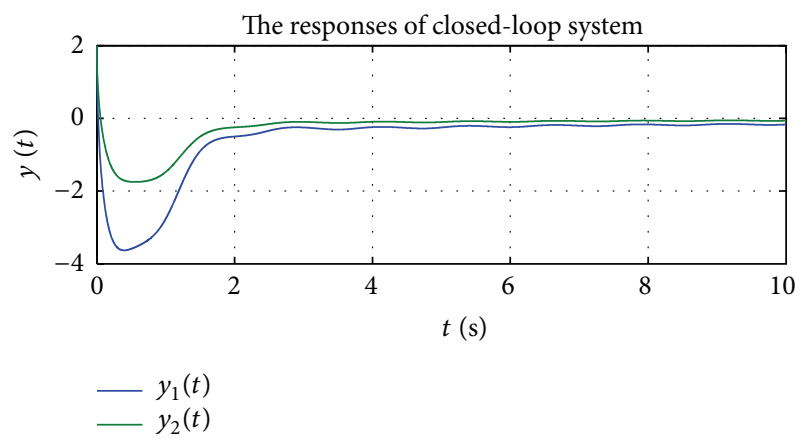

(b)

Figure 5: System responses of Example 5.

Example 3. Consider the system in (55) with $\alpha=1.4$.

Designing 1.4th order OFC as (3)-(5) and using the method in (43), one gets the equivalent system with 0.7 th order. By using the method for the case of $0<\alpha<1$, one gets the following feasible OFCs:

$$
\begin{gathered}
u(t)=-12.6764 y(t) \\
\mathscr{D}^{1.4} u(t)=-310.3178 y(t)-35.7741 u(t), \\
\mathscr{D}^{1.4} z(t)=-\left[\begin{array}{ll}
1.1129 & 0.0579 \\
0.0579 & 1.1129
\end{array}\right] z(t)-\left[\begin{array}{l}
0.1489 \\
0.1489
\end{array}\right] y(t), \\
u(t)=\left[\begin{array}{ll}
0.8829 & 0.8829
\end{array}\right] z(t)-12.7005 y(t) .
\end{gathered}
$$

Under the control of the obtained OFCs, one gives the eigenvalues distribution area of the equivalent closed-loop control system as shown in Figure 3.

One can observe from Figures 1, 2, and 3 that if the system (1) can be stabilized by OFC, no matter $0<\alpha<1$ or $1 \leq \alpha<$ 2 , we can get the needed controller via our approach.

Example 4. Consider the system as follows:

$$
\begin{gathered}
\mathscr{D}^{\alpha} x(t)=\left[\begin{array}{cc}
1 & -1 \\
0 & 0
\end{array}\right] x(t)+\left[\begin{array}{l}
1 \\
0
\end{array}\right] u(t), \\
y(t)=\left[\begin{array}{ll}
1 & 1
\end{array}\right] x(t) .
\end{gathered}
$$

If we design the static OFC as

$$
u(t)=K y(t)
$$

then one can get the eigenvalues of the closed-loop system matrix $A+B K C$ as

$$
\lambda= \begin{cases} \pm \sqrt{1-K}, & K \leq 1 \\ \pm \sqrt{K-1} i, & K>1\end{cases}
$$

To be obvious, when the order $\alpha>1$, no matter how we choose $K, A+B K C$ always has eigenvalue in unstable region. That is why we discuss the design of the dynamic OFCs in (3) and (5). Based on the method in this paper, we get the feasible dynamic OFCs as

$$
\begin{gathered}
\mathscr{D}^{1.1} u(t)=1.1791 y(t)-1.3799 u(t), \\
\mathscr{D}^{1.1} z(t)=-2.0121 z(t)+0.9878 y(t), \\
u(t)=2.1237 z(t)-1.0649 y(t) .
\end{gathered}
$$

From the results in Figure 4, we can obtain that the system in (59) can be stabilized by the obtained dynamic OFCs.

Example 5. Consider the uncertain unstable system

$$
\begin{aligned}
D^{\alpha} x(t)= & {\left[A_{0}+D F(t) E_{A}\right] x(t) } \\
& +\left[B_{0}+D F(t) E_{B}\right] u(t), \\
& y(t)=C x(t)
\end{aligned}
$$

with

$$
\begin{gathered}
A_{0}=\left[\begin{array}{lll}
1 & 1 & 0 \\
0 & 3 & 0 \\
0 & 0 & 2
\end{array}\right], \quad B_{0}=\left[\begin{array}{ll}
0 & 0 \\
1 & 0 \\
0 & 1
\end{array}\right], \quad D=\left[\begin{array}{l}
0.1 \\
0.2 \\
0.1
\end{array}\right], \\
E_{A}=\left[\begin{array}{lll}
0.1 & -0.1 & 0.2
\end{array}\right], \quad E_{B}=\left[\begin{array}{ll}
0.1 & 0.1
\end{array}\right], \\
F(t)=\sin (5 t), \quad C=\left[\begin{array}{lll}
1 & 2 & 1 \\
0 & 1 & 0
\end{array}\right], \quad \alpha=0.5 .
\end{gathered}
$$

Based on Theorem 16, one can get the static OFC

$$
u(t)=\left[\begin{array}{cc}
5.8640 & -13.0491 \\
-5.4278 & 8.4470
\end{array}\right] y(t),
$$

along with the virtual state-feedback control gain

$$
K_{0}=\left[\begin{array}{lll}
-3.0696 & -3.6873 & -0.0855 \\
-0.0194 & -0.0009 & -2.7295
\end{array}\right]
$$

The initial state is assumed as $x(0)=\left[\begin{array}{lll}1 & 2 & -3\end{array}\right]^{T}$. Figure 5 shows the output signal of the open-loop system 


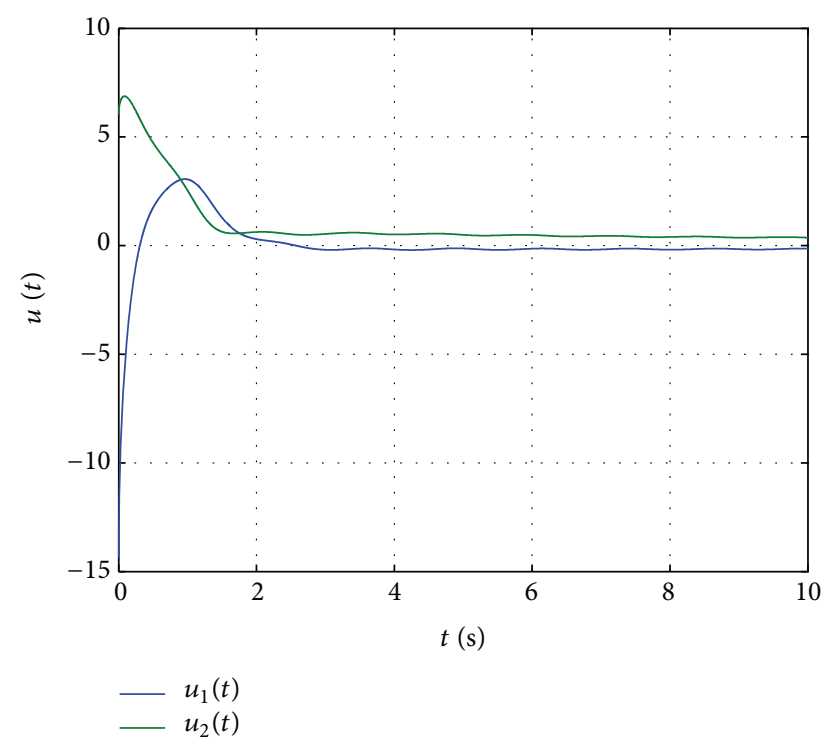

FIGURE 6: Control input of Example 5.

and the closed-loop system, respectively. The corresponding control input is given in Figure 6. From the simulation results, one can conclude that the proposed method can easily obtain OFC which is able to stabilize such uncertain fractional order system.

\section{Conclusions}

In this paper, the methods of designing general OFC for FOSs with the order $0<\alpha<2$ have been investigated. For the case of $0<\alpha<1$, LMI-based sufficient conditions for static/dynamic OFC design are proposed. Based on the equivalence transformation, the related results are generalized to the systems with $1 \leq \alpha<2$. Compared with existing results, the new proposed approaches require fewer decision variables and have less restrictions conditions which are helpful for reducing the conservatism of the obtained results. The numerical examples have shown the effectiveness of the proposed design methods. It is believed that the approaches provide a new avenue to solve such problem.

\section{Conflict of Interests}

The authors declare that there is no conflict of interests regarding the publication of this paper.

\section{Acknowledgments}

The authors would like to thank the Associate Editor and the anonymous reviewers for their keen and insightful comments which greatly improved the contents and the presentation.

\section{References}

[1] C. A. Monje, Y. Q. Chen, B. M. Vinagre, D. Xue, and V. Feliu, Fractional-Order Systems and Controls: Fundamentals and Applications, Springer, London, UK, 2010.
[2] S. Das, Functional Fractional Calculus, Springer, Berlin, Germany, 2011.

[3] Z. Liao, Z. T. Zhu, S. Liang, C. Peng, and Y. Wang, "Subspace identification for fractional order hammerstein systems based on instrumental variables," International Journal of Control, Automation and Systems, vol. 10, no. 5, pp. 947-953, 2012.

[4] A. M. Lopes and J. A. T. Machado, "Root locus practical sketching rules for fractional-order systems," Abstract and Applied Analysis, vol. 2013, Article ID 102068, 14 pages, 2013.

[5] M. D. Li, D. H. Li, J. Wang, and C. Zhao, "Active disturbance rejection control for fractional-order system," ISA Transactions, vol. 52, no. 3, pp. 365-374, 2013.

[6] S. Liang, C. Peng, Z. Liao, and Y. Wang, "State space approximation for general fractional order dynamic systems," International Journal of System Science, 2013.

[7] Z. Liao, C. Peng, W. Li, and Y. Wang, "Robust stability analysis for a class of fractional order systems with uncertain parameters," Journal of the Franklin Institute, vol. 348, no. 6, pp. 11011113, 2011.

[8] N. I. Mahmudov, R. Sakthivel, and P. Revathi, "Asymptotic stability of fractional stochastic neutral differential equations with infinite delays," Abstract and Applied Analysis, vol. 2013, Article ID 769257, 9 pages, 2013.

[9] S. Liang, C. Peng, and Y. Wang, "Improved linear matrix inequalities stability criteria for fractional order systems and robust stabilization synthesis: the $0<\alpha<1$ case," Control Theory \& Applications, vol. 30, no. 4, pp. 531-535, 2013.

[10] I. N'Doye, M. Darouach, M. Zasadzinski, and N.-E. Radhy, "Robust stabilization of uncertain descriptor fractional-order systems," Automatica, vol. 49, no. 6, pp. 1907-1913, 2013.

[11] J. Yuan, B. Shi, and W. Q. Ji, "Adaptive sliding mode control of a novel class of fractional chaotic systems," Advances in Mathematical Physics, vol. 2013, Article ID 576709, 13 pages, 2013.

[12] C. L. Li, K. L. Su, J. Zhang et al., "Robust control for fractionalorder four-wing hyperchaotic system using LMI," Optik International Journal for Light and Electron Optics, vol. 124, no. 22, pp. 5807-5810, 2013.

[13] M. M. Asheghan, S. S. Delshad, M. T. H. Beheshti, and M. S. Tavazoei, "Non-fragile control and synchronization of a new fractional order chaotic system," Applied Mathematics and Computation, vol. 222, no. 1, pp. 712-721, 2013.

[14] S. C. Tong, Y. M. Li, and P. Shi, "Observer-based adaptive fuzzy backstepping output feedback control of uncertain MIMO pure-feedback nonlinear systems," IEEE Transactions on Fuzzy Systems, vol. 20, no. 4, pp. 771-785, 2013.

[15] S. B. Attia, S. Salhi, and M. Ksouri, "Static switched output feedback stabilization for linear discrete-time switched systems," International Journal of Innovative Computing, Information and Control, vol. 8, no. 5, pp. 3203-3213, 2012.

[16] J. Wu, H. R. Karimi, and P. Shi, "Network-based $H_{\infty}$ output feedback control for uncertain stochastic systems," Information Sciences, vol. 232, pp. 397-410, 2013.

[17] L. G. Wu, W. X. Zheng, and H. J. Gao, "Dissipativity-based sliding mode control of switched stochastic systems," IEEE Transactions on Automatic Control, vol. 58, no. 3, pp. 785-791, 2013.

[18] X. Su, P. Shi, L. Wu, and Y. D. Song, "A novel control design on discrete-time Takagi-Sugeno fuzzy systems with time-varying delays," IEEE Transactions on Fuzzy Systems, vol. 21, no. 4, pp. 655-671, 2013.

[19] Y. He and Q. G. Wang, "An improved ILMI method for static output feedback control with application to multivariable PID 
control," IEEE Transactions on Automatic Control, vol. 51, no. 10, pp. 1678-1683, 2006.

[20] R. N. Yang, G. P. Liu, P. Shi, and C. Thomas, "Predictive output feedback control for networked control systems," IEEE Transactions on Industrial Electronics, vol. 61, no. 1, pp. 512-520, 2014.

[21] J. X. Dong and G. H. Yang, "Static output feedback control synthesis for linear systems with time-invariant parametric uncertainties," IEEE Transactions on Automatic Control, vol. 52, no. 10, pp. 1930-1936, 2007.

[22] J. B. Qiu, G. Feng, and H. J. Gao, “Observer-based piecewise affine output feedback controller synthesis of continuous-time TS fuzzy affine dynamic systems using quantized measurements," IEEE Transactions on Fuzzy Systems, vol. 20, no. 6, pp. 1046-1062, 2012.

[23] M. Chadli and T. M. Guerra, "LMI solution for robust static output feedback control of Takagi-Sugeno fuzzy models," IEEE Transactions on Fuzzy Systems, vol. 20, no. 6, pp. 1060-1065, 2012.

[24] D. M. Zhang, X. G. Wang, and L. Meng, "Consensus problems for high-order LTI systems: a decentralized static output feedback method," International Journal of Innovative Computing, Information and Control, vol. 9, no. 5, pp. 2143-2154, 2013.

[25] S. M. Saadni, M. Chaabane, and D. Mehdi, "Robust stability and stabilization of a class of singular systems with multiple timevarying delays," Asian Journal of Control, vol. 8, no. 1, pp. 1-11, 2006.

[26] L. G. Wu, X. J. Su, and P. Shi, "Output feedback control of Markovian jump repeated scalar nonlinear systems," IEEE Transactions on Automatic Control, vol. 59, no. 1, pp. 199-204, 2013.

[27] Y. Fang and W. Jiang, "Output feedback stabilization for a type of fractional-order systems with delay," Information and Control, vol. 42, no. 1, pp. 33-38, 2013.

[28] I. N'Doye, H. Voos, M. Darouach et al., " $H_{\infty}$ static output feedback control for a fractional-order glucose-insulin system," Fractional Differentiation and Its Applications, vol. 6, no. 1, pp. 266-271, 2013.

[29] Y. Wang, Y. H. Wei, M. Zhu, and P. Cheng, "A novel LMI approach for robust stabilization of uncertain fractional order systems," in Proceedings of the 32nd Chinese Control Conference (CCC '13), pp. 1475-1479, Xi'an, China, July 2013.

[30] Z. Q. Lou, "Static output feedback robust stability control for fractional linear systems," Microcomputer Applications, vol. 26, no. 8, pp. 11-14, 2010.

[31] P. Gahinet and P. Apkarian, "A linear matrix inequality approach to $H_{\infty}$ control," International Journal of Robust and Nonlinear Control, vol. 4, no. 4, pp. 421-448, 1994. 


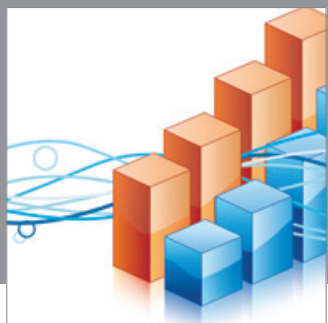

Advances in

Operations Research

mansans

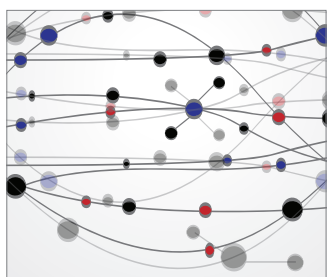

The Scientific World Journal
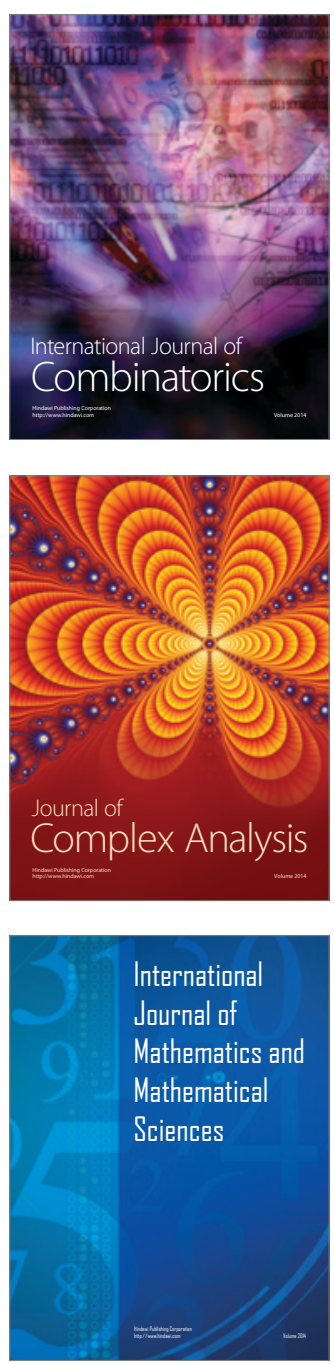
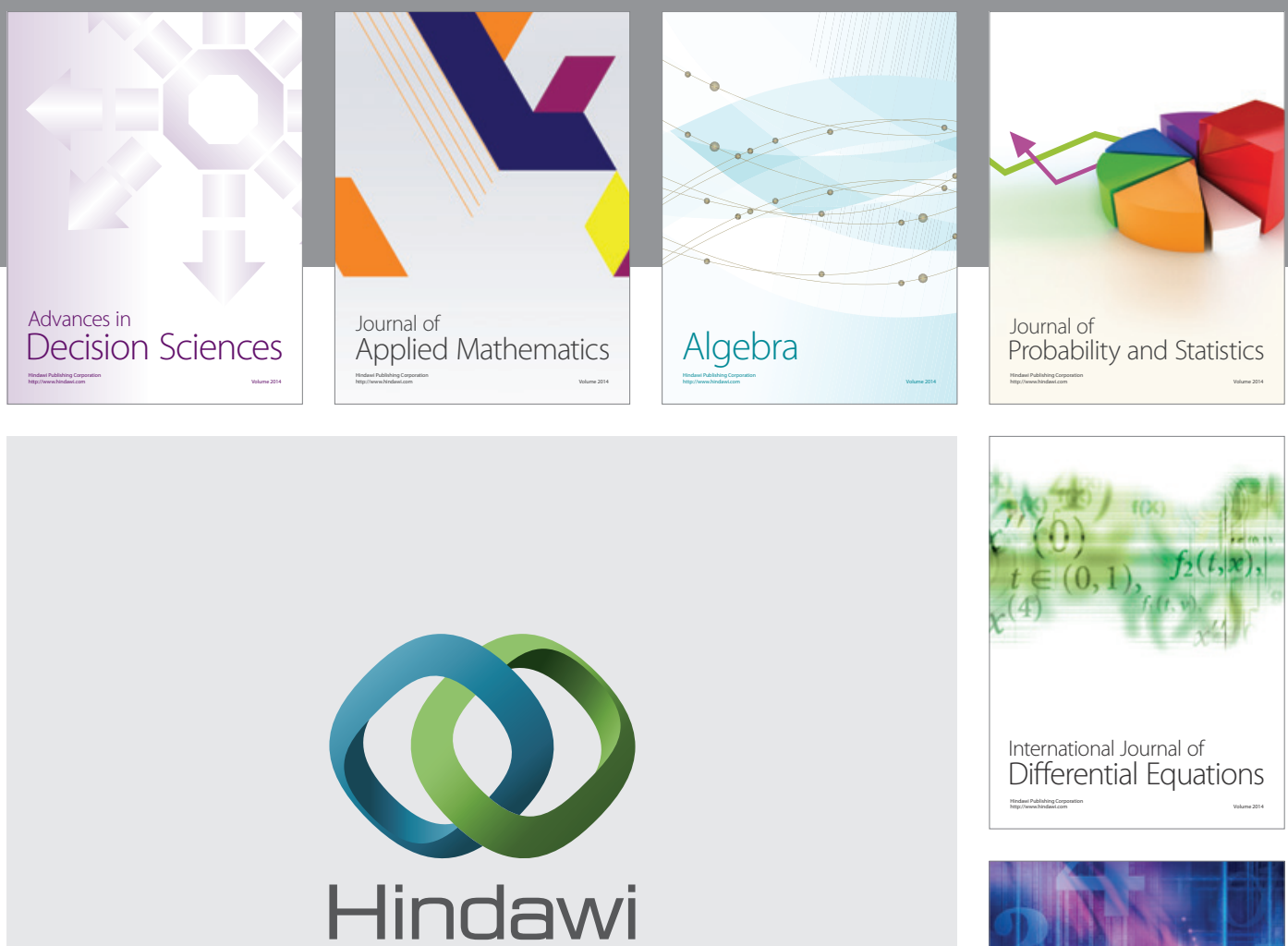

Submit your manuscripts at http://www.hindawi.com
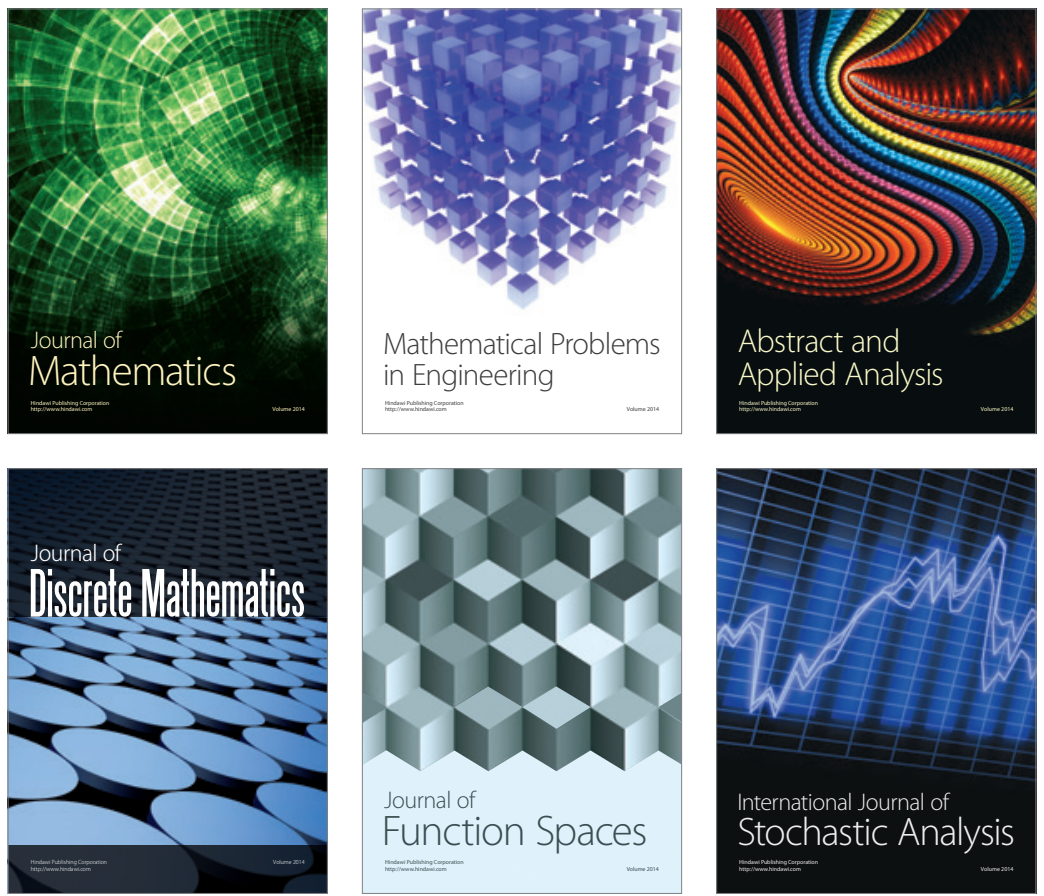

Journal of

Function Spaces

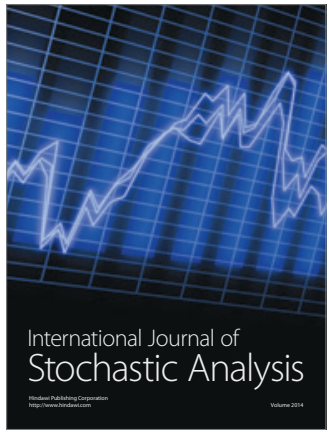

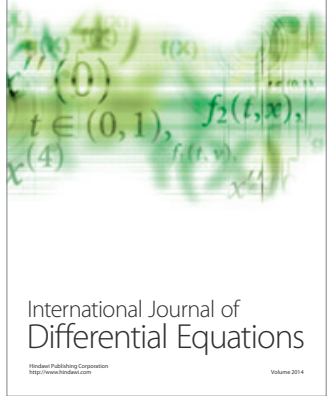
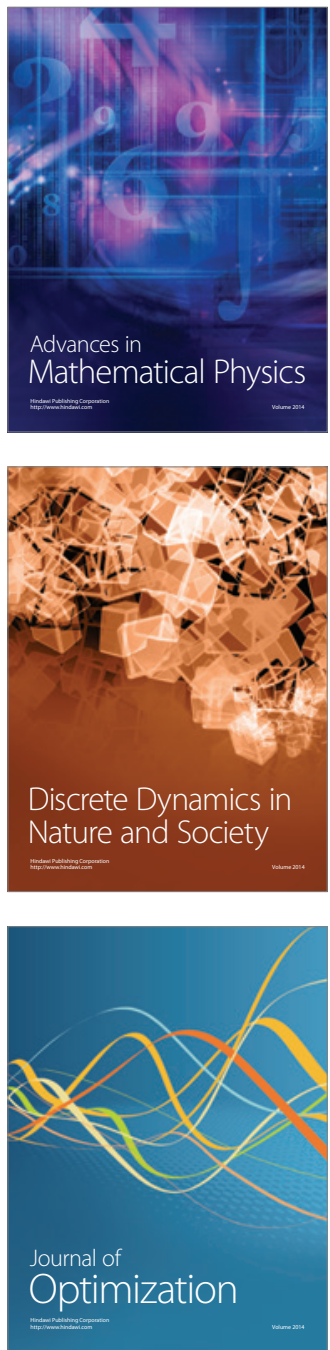